\title{
Detection Techniques for Diffusion-based Molecular Communication
}

\author{
Ignacio Llatser, Albert Cabellos-Aparicio, Massimiliano Pierobon, Student Member, IEEE, \\ and Eduard Alarcón, Member, IEEE
}

\begin{abstract}
Nanonetworks, the interconnection of nanosystems, are envisaged to greatly expand the applications of nanotechnology in the biomedical, environmental and industrial fields. However, it is still not clear how these nanosystems will communicate among them. This work considers a scenario of Diffusionbased Molecular Communication (DMC), a promising paradigm that has been recently proposed to implement nanonetworks. In a DMC network, transmitters encode information by the emission of molecules which diffuse throughout the medium, eventually reaching the receiver locations. In this scenario, a pulse-based modulation scheme is proposed and two techniques for the detection of the molecular pulses, namely, amplitude detection and energy detection, are compared. In order to evaluate the performance of DMC using both detection schemes, the most important communication metrics in each case are identified. Their analytical expressions are obtained and validated by simulation. Finally, the scalability of the obtained performance evaluation metrics in both detection techniques is compared in order to determine their suitability to particular DMC scenarios. Energy detection is found to be more suitable when the transmission distance constitutes a bottleneck in the performance of the network, whereas amplitude detection will allow achieving a higher transmission rate in the cases where the transmission distance is not a limitation. These results provide interesting insights which may serve designers as a guide to implement future DMC networks.
\end{abstract}

Index Terms-Diffusion-based molecular communication, signal detection, pulse-based modulation, nanonetworks.

\section{INTRODUCTION}

$\mathbf{N}$ ANOTECHNOLOGY, the study of systems within the nanometer scale, is a multidisciplinary field with potential applications in the biomedical [1], environmental [2] and industrial fields [3]. A nanosystem is the most basic functional unit, with a size ranging from one to a few hundred nanometers, able to perform simple tasks at the nanoscale, such as computing, data storage, sensing and actuation.

I. Llatser, A. Cabellos-Aparicio and E. Alarcón are with the Nanonetworking Center in Catalunya (N3Cat), Universitat Politècnica de Catalunya, Barcelona, Spain, e-mail: \{1latser,acabello $\} @$ ac.upc.edu, eduard.alarcon@upc.edu

M. Pierobon is with the Broadband Wireless Networking Laboratory, School of Electrical and Computer Engineering, Georgia Institute of Technology, Atlanta, Georgia 30332, USA, e-mail: maxp@ece.gatech.edu
However, despite the high potential of nanosystems, most of the envisaged applications of nanotechnology cannot be implemented by a single nanosystem, due to its reduced size and limited operation range. Nanonetworks [4], the interconnection of nanosystems, will allow cooperation and information sharing among them, making the realization of these applications possible. A sample application within the biomedical field enabled by nanonetworks would be an intelligent disease detection and targeted drug delivery system, constituted by an intra-body network of nanosensors and nanoactuators.

Several methods have been proposed to interconnect nanosystems by drawing inspiration from biology. Among them is calcium signaling [5], one of the most commonly used techniques for intra- and inter-cellular communication. Calcium signaling is based on the use of calcium ions $\left(\mathrm{Ca}^{2+}\right)$ to encode, transmit and decode information. Since calcium signaling has successfully been used by living cells for millions of years, it is regarded as one of the most promising techniques to implement communication among nanosystems in the short range [6], [7].

Calcium signaling and other communication techniques based on the exchange of molecules in a fluid environment can be characterized with an emerging model known as Diffusion-based Molecular Communication (DMC). Several authors have attempted to model the physical channel of DMC from a general perspective, without assuming any particular modulation or detection schemes [8], [9], [10]; while others have focused on determining the channel capacity of DMC from an information-theoretical point of view [11], [12], [13], [14].

However, to the best of our knowledge, the existing literature does not capture all the particular characteristics of DMC networks. For instance, the expected simplicity of nanosystems will prevent the use of complex modulations or signal detection schemes. Therefore, a framework that evaluates the performance of DMC networks while taking into account the uniqueness of their physical channel is needed to help designers of protocols and techniques for this networking model.

In this work, we consider a DMC network using a simple pulse-based modulation scheme which we recently proposed [15]. In this scenario, we identify two 
schemes for the detection of the transmitted molecular pulses: amplitude detection and energy detection. We first review our previous work [15], where the performance of DMC networks using amplitude detection was studied. With this purpose, quantitative expressions for relevant communication metrics, namely, the pulse delay, amplitude and width, were analytically derived, validated by simulation and compared to their equivalent in wireless electromagnetic (EM) communications.

As the main contribution of this work, we propose and analyze the mechanism of energy detection for DMC. In order to evaluate the performance of this novel detection technique, we identify two new relevant communication metrics in this scenario: the pulse energy and the pulse duration. For each of these metrics, we obtain analytical expressions and we validate them by simulation. Finally, we compare the scalability of the performance metrics corresponding to both signal detection mechanisms to their equivalent in wireless EM communications; we conclude that the suitability of each pulse detection technique will be determined by the average transmission distance in the DMC network.

The remainder of this paper is organized as follows. Section II describes the DMC scenario considered and the proposed pulse-based modulation scheme. Section III briefly presents N3Sim, a simulator for DMC which is later used to validate the obtained results. In Section IV, two detection methods for the proposed scenario are introduced, namely, amplitude detection and energy detection. Then, in Sections V and VI, we obtain analytical expressions of the relevant communication metrics in the amplitude detection and energy detection scenarios, respectively. Section VII compares the scalability of these metrics in both scenarios with their equivalent in wireless EM communications. Finally, Section VIII concludes the paper.

\section{Diffusion-Based Molecular COMmunication and Modulation Scheme}

A DMC network (shown in Fig. 1) is composed of a set of nanosystems which communicate through molecular diffusion in a fluid medium. Transmitter nanosystems encode the information to be sent into a molecular release pattern. The emitted molecules cause a variation in their local concentration, which propagates throughout the medium. Receivers are able to estimate the concentration of molecules in their neighborhood and, from this measurement, recover the release pattern of molecules and decode the transmitted information.

In a typical DMC scenario, the concentration of emitted molecules will be much lower than the concentration of the fluid molecules. For instance, in calcium signaling among cells, the extracellular concentration of calcium ions is in the millimolar range [16], while the concentration of water (the main component of the extracellular

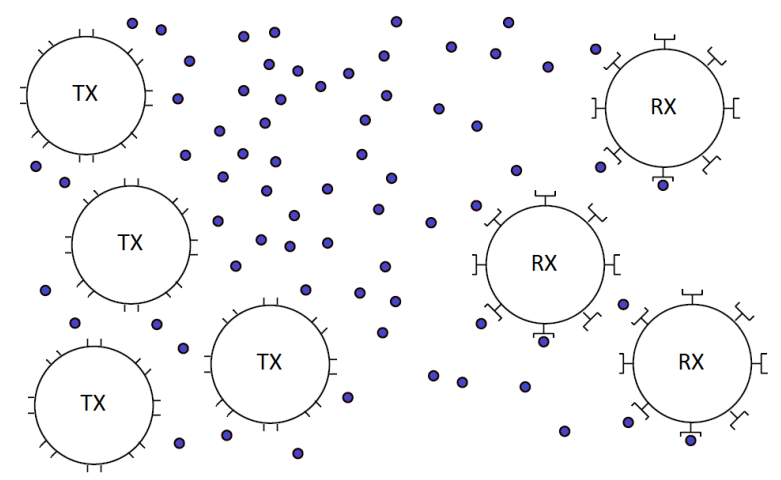

Fig. 1. Conceptual diagram of a DMC network. A group of transmitter nanosystems (left) emits molecules which propagate through molecular diffusion until they reach the receiver nanosystems (right).

fluid) is of 55.5 molar, more than 4 orders of magnitude higher. Under these conditions, the interaction among the emitted molecules (e.g., collisions and electrostatic forces) can be neglected, and the movement of each of the molecules released by a transmitter can therefore be modeled by means of Brownian motion [17]. Since the movement of each molecule is independent, molecular diffusion is characterized by Fick's laws of diffusion [18], with a homogeneous diffusion coefficient both in space and time. In this case, the diffusion equations are linear [19].

Furthermore, the expected simplicity of nanosystems will prevent the use of complex modulation schemes in DMC, such as OFDM or spread-spectrum techniques. For this reason, we recently proposed a simple pulsebased modulation particularly suited for DMC [15]. According to this scheme, whenever a transmitter nanosystem needs to communicate information to its neighbors (e.g., after it detects an infectious virus [20]), it instantaneously releases a pulse of molecules. This creates a spike in the molecular concentration at the transmitter location, which then propagates throughout the space. The propagation of this pulse can be analytically modeled by solving Fick's laws of diffusion. If the transmitter releases $Q$ molecules at the time instant $t=0$, the molecular concentration at any point in space is given by [21]:

$$
c(r, t)=\frac{Q}{(4 \pi D t)^{3 / 2}} e^{-r^{2} / 4 D t}
$$

where $D$ is the diffusion coefficient of the medium, $t$ is time and $r$ is the distance from the transmitter location.

We denote this expression as the pulse equation. Fig. 2 shows a graphical representation of the molecular concentration measured by a receiver as a function of time, where the dashed blue line corresponds to the analytical result given by (1) and the red dots are simulation results, obtained using N3Sim (see Section III). In this example, the number of transmitted molecules is set to $Q=5 \cdot 10^{5}$, 


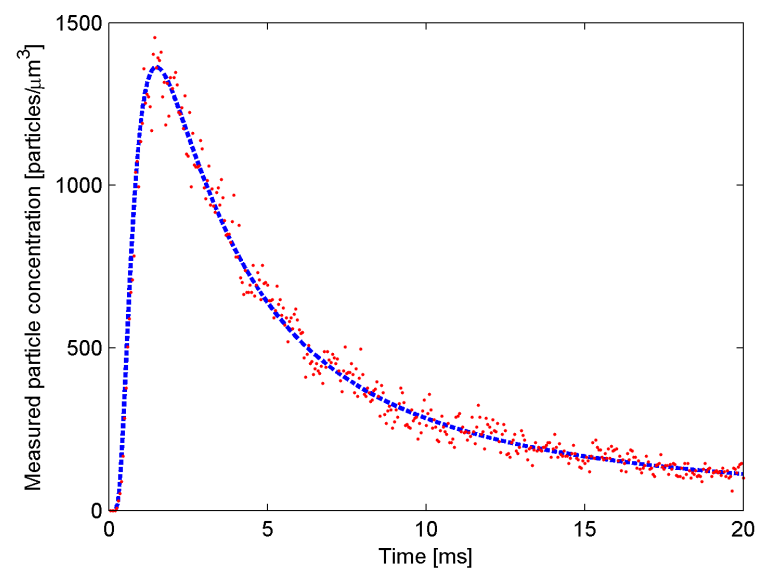

Fig. 2. Molecular concentration as a function of time at a distance of $3 \mu \mathrm{m}$ from a transmitter, after a pulse of $5 \cdot 10^{5}$ molecules has been transmitted. The dashed blue line shows the analytical expression and the red dots correspond to simulation results.

the transmission distance is $r=3 \mu \mathrm{m}$ and the diffusion coefficient is $D=1 \mathrm{~nm}^{2} / \mathrm{ns}$, similar to the diffusion coefficient of ionic calcium in cytoplasm [22]. The transmitter is modeled as a point-wise source, whereas the receiver is represented by a sphere with a radius of $0.4 \mu \mathrm{m}$ which is able to measure the average molecular concentration in its interior.

We observe that the concentration measured by the receiver is initially zero and it quickly increases until reaching its maximum. Thereafter, the molecular concentration slowly decreases over time, forming a long tail due to the effect of diffusion. The difference between the expression of the pulse equation and the simulation results shows the presence of diffusion noise, which appears as a result of the stochastic nature of Brownian motion [9]. The diffusion noise represents the main limitation in the minimum molecular concentration that can be detected by a receiver, i.e., the receiver sensitivity. The expression of the diffusion noise $n(r, t)$ has zero mean and its standard deviation is given by [9]:

$$
\sigma[n(r, t)]=\sqrt{\frac{c(r, t)}{(4 / 3) \pi \rho^{3}}}
$$

where $\rho$ is the radius of the spherical receiver. We clearly observe that the diffusion noise has an inverse dependence on the receiver size: the larger the receiver is, the lower the diffusion noise will be.

As we will see next, the pulse equation allows to model important communication metrics in a DMC environment, such as the delay, attenuation and energy of transmitted signals, which present fundamental differences with respect to wireless EM communications and prove the uniqueness of the physical channel in DMC. Since the pulse equation considers the average value of the molecular concentration, this analysis will not be affected by the previously-described diffusion noise, which has zero mean.

\section{N3Sim, A SimUlator FOR MOLECUlAR COMMUNICATION}

In order to validate the analytical results derived in this work, we use the open-source simulation framework N3Sim, which we previously developed [23], [24]. This tool allows the simulation of DMC networks moleculeby-molecule, with multiple transmitters and receivers suspended in a 3-dimensional fluid medium. Each transmitter is modeled as a point-wise nanosystem with a fixed location. Transmitters encode the information by releasing molecules into the medium with a userspecified pattern. The emitted molecules move according to Brownian motion, as a result of collisions with the smaller fluid molecules. Finally, receivers are modeled as spherical nanosystems which are able to measure the number of molecules in their surrounding volume, thus estimating their local concentration. The received information is decoded from this local concentration estimate.

The main output of N3Sim is the concentration as a function of time measured by each of the receivers. The results obtained by N3Sim allow the evaluation of the molecular diffusion process as well as the measurement of the diffusion noise in a DMC network. The interested reader can find more details about N3Sim in our previous work [23] and on our website [24].

\section{Detection Methods for DMC with a Pulse-BASEd Modulation}

We propose two methods for receivers to detect the transmitted pulses in a DMC network. First, using amplitude detection, receivers measure the variation of the local concentration of molecules over time. Then, the measured signal is interpreted as a bit " 1 " if its maximum concentration is over a given threshold, and as a bit " 0 " otherwise. The value of this threshold should be selected as a function of the expected amplitude of the received signal.

An alternative technique for pulse detection in DMC is energy detection. Making an analogy to EM communications, where the pulse energy is calculated as the integral of its power over time, the pulse energy in DMC is defined as the integral of the molecular concentration over time. Using this method, a receiver measures the energy of the molecular signal. The received signal is then interpreted as a bit " 1 " if its energy is over a given threshold, and as a bit " 0 " otherwise. A simple way for receivers to measure the pulse energy would be by accumulating the number of molecules received during the pulse duration.

Considering the pulse-based modulation scheme introduced in Section II, we explore next the characteristics 
of the physical channel of DMC using both detection methods by analyzing the pulse equation (1). With this purpose, we identify several metrics that will allow the assessment of the communication performance of DMC in different scenarios. In particular, we first obtain analytical expressions for these metrics and then validate them by simulation. In order to make this analysis as generalpurpose and technology-agnostic as possible, we do not set any constraints on the actual physical implementation of transmitters and receivers, nor on the size and type of the emitted molecules.

\section{Amplitude Detection}

We analyze next three relevant communication metrics in a DMC scenario using amplitude detection. First, we consider the pulse delay, which will determine the communication delay between the transmission and reception of molecular signals. Next, we analyze the pulse amplitude, which, as previously mentioned, will have a key impact on the detection of molecular signals. Finally, we evaluate the pulse width, which will represent the main constraint for the achievable transmission rate using this technique.

\section{A. Pulse Delay}

In order to find an expression for the pulse delay, we compute the time instant for which the pulse equation reaches its global maximum. As we observe in Fig. 2, this function has only one local maximum, which is also its global maximum. We can therefore compute the position of this maximum by taking the time derivative of the pulse equation and finding the time instant at which it is equal to zero:

$$
\frac{d c(r, t)}{d t}=\frac{d}{d t} \frac{Q e^{-r^{2} / 4 D t}}{(4 \pi D t)^{3 / 2}}=0 .
$$

From this equation, by isolating the variable $t$ we can obtain the time at which the pulse has its maximum. This time can be interpreted as the pulse delay $t_{d}$ :

$$
t_{d}=\frac{r^{2}}{6 D}
$$

Note that the pulse delay is inversely proportional to the diffusion coefficient $D$. Hence, the higher the diffusion coefficient, the faster the molecular pulses will propagate.

In order to validate this result, we simulate the transmission of a pulse of $5 \cdot 10^{5}$ molecules using N3Sim. The diffusion coefficient is set to $D=1 \mathrm{~nm}^{2} / \mathrm{ns}$ and the local molecular concentration is measured at distances from 1 to $4.5 \mu \mathrm{m}$, at intervals of $0.5 \mu \mathrm{m}$. Fig. 3 shows a comparison between the analytical expression of the pulse delay (dashed blue line) and the averaged results obtained with N3Sim after 30 simulation runs with $99 \%$ confidence intervals. We will use these same simulation conditions throughout this paper, unless otherwise stated.

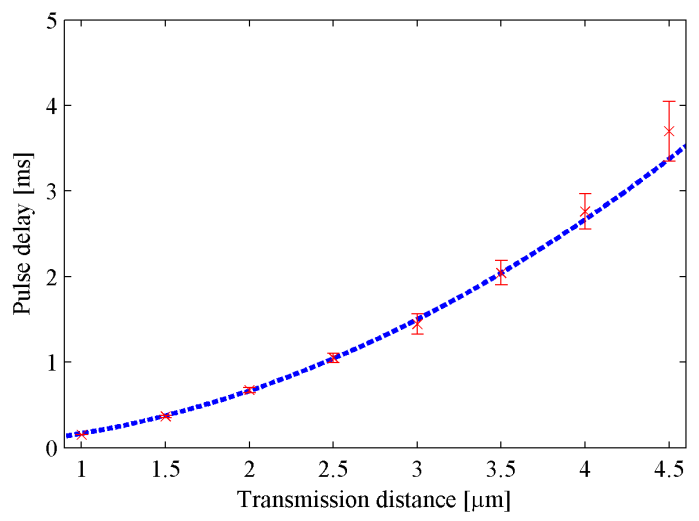

Fig. 3. Plot of the pulse delay as a function of the transmission distance. The dashed blue line corresponds to the analytical expression and the red crosses show the simulation results with $99 \%$ confidence intervals.

\section{B. Pulse Amplitude}

It is also worth investigating the variation of the pulse amplitude over space, which may be interpreted as the channel attenuation. We obtain this amplitude by evaluating the pulse equation at the time instant at which the pulse reaches its maximum value, which we have previously found in (4):

$$
c_{\max }=\left.c(r, t)\right|_{t=t_{d}}=\left(\frac{3}{2 \pi e}\right)^{3 / 2} \frac{Q}{r^{3}} .
$$

It is interesting to note that, as opposed to the pulse delay, the pulse amplitude is independent from the diffusion coefficient. In consequence, the diffusion coefficient of the medium will have no effect on the attenuation of the molecular pulses throughout space.

As previously, we validate this result by means of simulation. Considering a pulse transmission with the same characteristics as used to validate the pulse delay, we measure the pulse amplitude as a function of the transmission distance. Fig. 4 shows a comparison of the analytical expression and the simulation results, which confirms the correctness of (5).

\section{Pulse Width}

Another important metric in a scenario of DMC with amplitude detection is the pulse width, since it will be the main constraint on the achievable transmission rate. As it is usually done in EM communications, we compute the pulse width at the $50 \%$ level, i.e., the time interval at which the pulse has an amplitude greater than half of its maximum value:

$$
c(r, t)=\frac{Q}{(4 \pi D t)^{3 / 2}} e^{-r^{2} / 4 D t}=\frac{c_{\max }}{2}=\frac{1}{2}\left(\frac{3}{2 \pi e}\right)^{3 / 2} \frac{Q}{r^{3}} .
$$




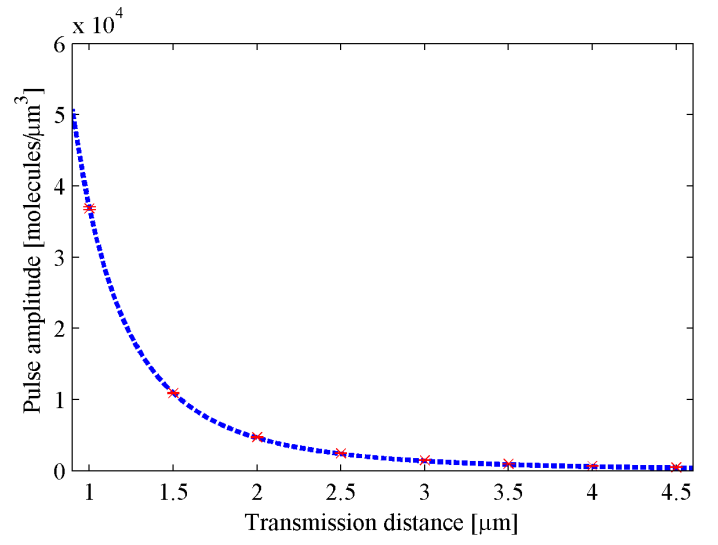

Fig. 4. Plot of the pulse amplitude as a function of the transmission distance. The dashed blue line corresponds to the analytical expression and the red crosses show the simulation results with $99 \%$ confidence intervals.

We obtain the following expression by isolating the time variable:

$$
t=-\frac{r^{2}}{6 D W\left(-\frac{1}{2^{2 / 3} e}\right)}
$$

where $W$ is the Lambert $\mathrm{W}$ function [25]. This equation has two solutions, corresponding to the two time instants at which the pulse amplitude is equal to half of its maximum value. These instants are given by:

$$
t_{1}=\frac{0.0728}{D} r^{2}, \quad t_{2}=\frac{0.5229}{D} r^{2} .
$$

Finally, we can obtain the expression of the pulse width $t_{w}$ by subtracting these two instants:

$$
t_{w}=t_{2}-t_{1}=\frac{0.4501}{D} r^{2} .
$$

In this case, as it happened with the pulse delay, the pulse width is inversely proportional to the diffusion coefficient of the medium. Therefore, the larger the diffusion coefficient is, the narrower the received pulses will be.

As before, we validate the obtained expression with N3Sim, using the same parameters as in the previous sections. Fig. 5 shows that the simulation results are close to the values of the analytical expression, which confirms the validity of (9).

\section{ENERGY DETECTION}

Let us now study the physical channel of DMC in the case that the receivers implement energy detectors to decode the transmitted molecular pulses. In this scenario of energy detection, some of the metrics that we have identified in the amplitude detection case (such as the pulse delay) are still meaningful, but new communication metrics will become relevant as well.

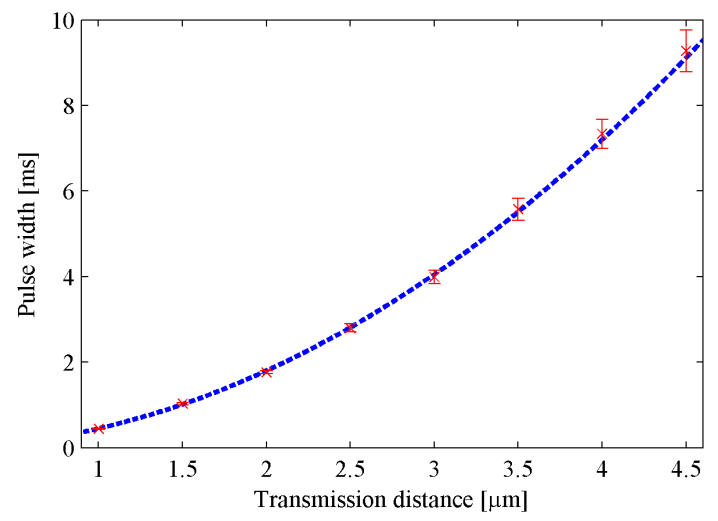

Fig. 5. Plot of the pulse width as a function of the transmission distance. The dashed blue line corresponds to the analytical expression, and the red crosses show the simulation results with $99 \%$ confidence intervals.

In particular, the pulse amplitude determines the receiver sensitivity (i.e., the ability to distinguish whether the received signal represents a bit " 0 " or " 1 ") in the amplitude detection case. In an energy detection scenario, however, in order to evaluate the receiver sensitivity we need to analyze a new communication metric, namely, the pulse energy.

Furthermore, the achievable transmission rate in DMC using amplitude detection is limited by the pulse width. In order to see this limitation intuitively, let us consider the case where a bit stream is encoded in a train of molecular pulses. In this case, the minimum separation between the transmitted pulses needs to be approximately equal to the pulse width at the receiver, so that consecutive pulses can be distinguished and the information can be correctly decoded (assuming that the transmitter and receiver are synchronized). The achievable transmission rate in this scenario will thus correspond approximately to the inverse of the pulse width at the receiver location. As we will see next, in an energy detection scenario, we need to define the pulse duration as the communication metric that will determine the maximum achievable transmission rate.

In what follows, analytical expressions for the two communication metrics that have just been identified, namely, the pulse energy and the pulse duration, are obtained. Further, they are validated by simulation and their impact in the performance of DMC using energy detection is discussed.

\section{A. Pulse Energy}

The most important metric to consider in the energy detection case is the pulse energy; indeed, energy detectors will decode the received signals by measuring their energy and comparing it to a threshold, which will be a function of the expected pulse energy. Since the pulse energy in DMC is defined as the temporal sum of 


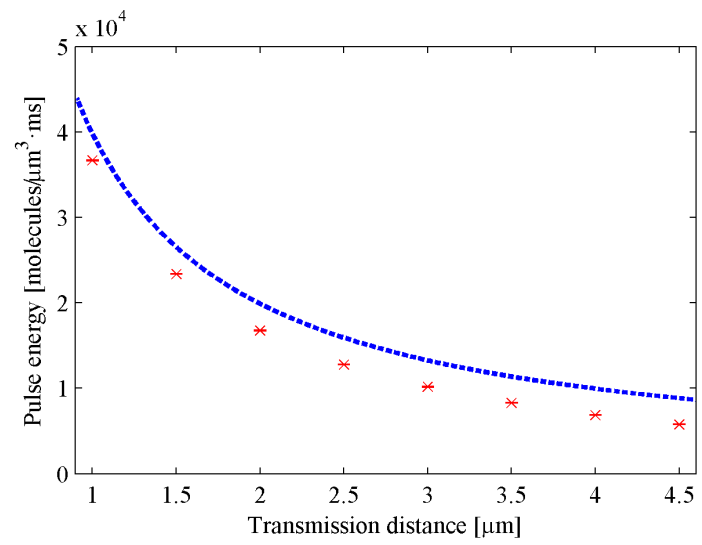

Fig. 6. Plot of the pulse energy as a function of the transmission distance. The dashed blue line corresponds to the analytical expression, and the red crosses show the simulation results with $99 \%$ confidence intervals.

its molecular concentration, the energy of a molecular pulse can be computed by integrating the pulse equation over time:

$E_{p}=\int_{0}^{\infty} c(r, t) \mathrm{d} t=\int_{0}^{\infty} \frac{Q}{(4 \pi D t)^{3 / 2}} e^{-\frac{r^{2}}{4 D t}} \mathrm{~d} t=\frac{Q}{4 \pi D r}$

In order to validate this result, as done in the previous section, the transmission of a pulse of $5 \cdot 10^{5}$ molecules is simulated using N3Sim. Fig. 6 shows a comparison between the analytical expression of the pulse energy (dashed blue line) and the averaged results after 30 simulation runs with $99 \%$ confidence intervals. In this case, we observe that, even though the analytical and simulation results show a similar depencence with respect to the transmission distance, the values obtained by simulation are lower than the analytically-obtained pulse energy. The reason for this difference is that, since molecular pulses have an infinite tail due to the effect of diffusion (see Fig. 2), a receiver would need an infinite time to measure the exact value of the pulse energy, i.e., that derived by solving the improper integral in (10). Since, in a practical scenario, receivers will only have a finite time to measure the pulse energy (e.g., the performed simulations have a duration of $50 \mathrm{~ms}$ ), the pulse energy measured by a receiver will always have a lower value than the energy predicted by the analytical expression.

\section{B. Pulse Duration}

As opposed to the pulse width in an amplitude detection scenario [15], it will be the pulse duration which will determine the achievable transmission rate in DMC using energy detection. As we have just observed, if the pulse duration were defined as the time needed by a receiver to measure the pulse energy $E_{p}$, it would have an infinite value. Instead, in order to allow receivers to

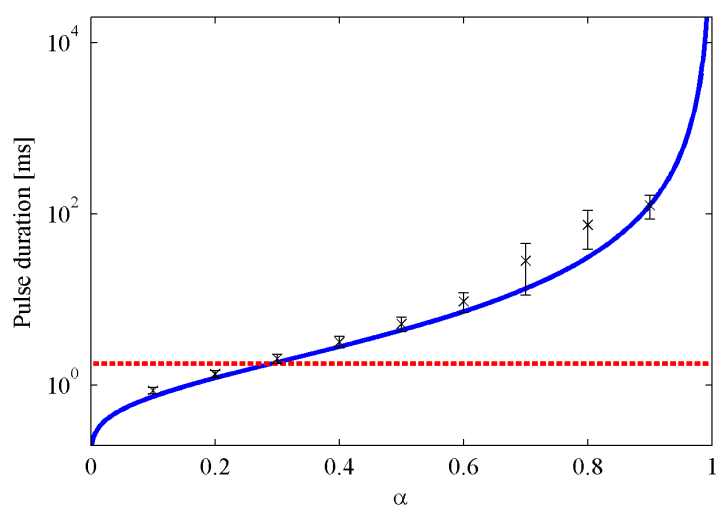

Fig. 7. Semi-log plot of the pulse duration as a function of the fraction of the pulse energy required to detect a pulse $\alpha$. The solid blue line corresponds to the analytical expression, and the black crosses show the simulation results with $99 \%$ confidence intervals. The pulse width in an amplitude detection scenario (dashed red line) is shown for the sake of comparison.

detect molecular pulses in a finite amount of time, we define the pulse duration as the time interval from the pulse transmission until the instant when a fraction $\alpha$ of the pulse energy has reached the receiver location. In other words, the pulse duration is the time needed to measure an energy $\alpha E_{p}$, where $0 \leq \alpha \leq 1$. We compute the pulse duration $t_{D}$ by solving the integral equation:

$$
\int_{0}^{t_{D}} c(r, t) \mathrm{d} t=\alpha E_{p}=\alpha \frac{Q}{4 \pi D r}
$$

which can be analytically solved and yields the following solution for the pulse duration:

$$
t_{D}=\frac{r^{2}}{4 D \operatorname{erfc}^{-1}(\alpha)^{2}}
$$

where $\mathrm{erfc}^{-1}$ is the inverse complementary error function [26]. Fig. 7 shows a semi-log plot of the pulse duration calculated by (12), as a function of $\alpha$, in a scenario of energy detection with a transmission distance $r=2 \mu \mathrm{m}$. As usual, the analytical results (solid blue line) are validated by simulation (black crosses). The pulse duration is also compared to the equivalent metric in the amplitude detection case, the pulse width (dashed red line), as obtained from (9).

As expected, the pulse duration increases monotonically with $\alpha$, the fraction of the pulse energy considered, and it tends to infinity when $\alpha$ approaches one: $\lim _{\alpha \rightarrow 1} t_{D}=\infty$. Therefore, from the point of view of the pulse duration, lower values of $\alpha$ yield a better performance. However, selecting a too low value of $\alpha$ might cause detection errors due to diffusion noise [9]. Therefore, a realistic value for $\alpha$ will probably be between 0.5 and 0.8 . In the amplitude detection case, the pulse width is $t_{w}=1.8 \mathrm{~ms}$. When comparing both scenarios, we observe that the pulse duration in the case 


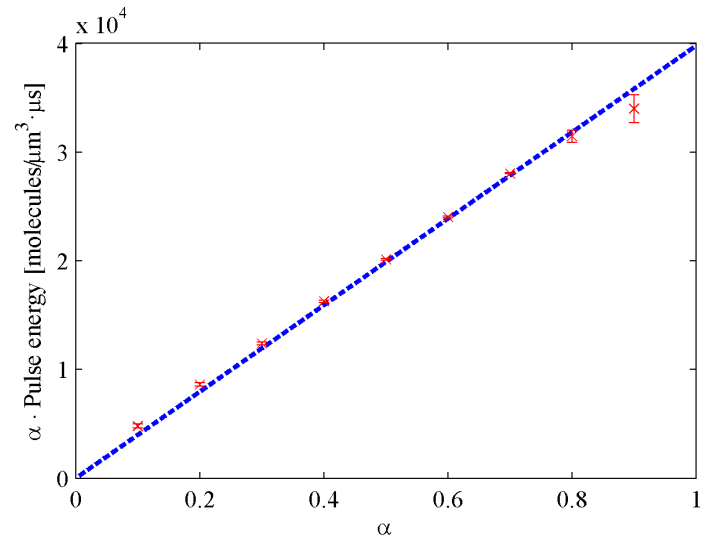

Fig. 8. Plot of the required energy to detect a pulse, as a function of the fraction of the pulse energy required to detect a pulse $\alpha$. The dashed blue line corresponds to the analytical expression, and the red crosses show the simulation results with $99 \%$ confidence intervals.

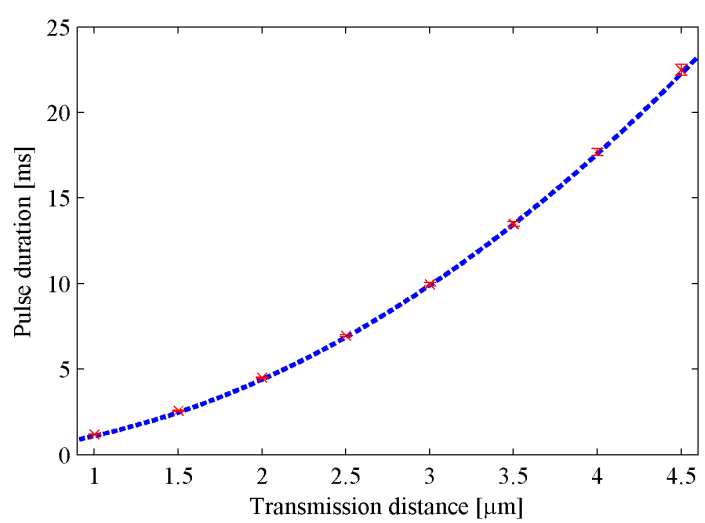

Fig. 9. Plot of the pulse duration as a function of the transmission distance. The dashed blue line corresponds to the analytical expression, and the red crosses show the simulation results with $99 \%$ confidence intervals.

of energy detection is lower than the pulse width for $\alpha<0.292$, and it is higher otherwise. In particular, for realistic values of $\alpha$, the pulse duration will be significantly higher than the pulse width when amplitude detection is used. For instance, for energy detection with $\alpha=0.7$, the pulse duration is almost one order of magnitude higher than the pulse width.

Furthermore, Fig. 8 shows the required energy to detect a pulse $\alpha E_{p}$, as a function of $\alpha$. The analytical results are obtained from (11) with a pulse of $Q=$ $10^{3}$ molecules transmitted to a distance $r=2 \mu \mathrm{m}$, whereas the simulations consider the energy measured by a receiver during the pulse duration $t_{D}$, as obtained from (12). Similarly, Fig. 9 shows the pulse duration as a function of the transmission distance. In both cases, a very good agreement between the analytical results (blue dashed lines) and the simulations (red crosses) is observed, thus validating the derived expressions.

\section{Scalability of Detection Techniques IN DMC}

It is of key interest to evaluate the scalability of the previously found communication metrics with respect to the transmission distance. We next compare the results obtained in both the amplitude detection and energy detection cases in DMC to their equivalent in wireless EM communications.

Starting with the amplitude detection scenario, as we observe in (4), the pulse delay is proportional to the square of the transmission distance, namely, $t_{d}=$ $\Theta\left(r^{2}\right)$. This is due to the peculiarities of the Brownian motion underlying the diffusion process, which is fundamentally different from the constant wave propagation speed observed in EM communications. In the latter case, the propagation delay is equal to the transmission distance divided by the wave propagation speed, namely, $t_{d}=\Theta(r)$.

Equation (5) shows that the amplitude of a molecular pulse is inversely proportional to the third power of the transmission distance, i.e., $c_{\max }=\Theta\left(1 / r^{3}\right)$. This dependence shows again a difference with respect to the behavior of waves in wireless EM communications, for which the free-space path loss is $A=\left(\frac{4 \pi r f}{c}\right)^{2}$, where $f$ is the signal frequency and $c$ the speed of light. Therefore, if we ignore fading, the amplitude of EM pulses propagating in free space decreases proportionally to the square of the transmission distance, namely, $c_{\max }=\Theta\left(1 / r^{2}\right)$, in contrast with the behavior observed in a DMC scenario.

Furthermore, according to (9), the dependence of the pulse width in DMC on the transmission distance is $t_{w}=\Theta\left(r^{2}\right)$. Again, there is a clear difference with the wireless EM channel, for which the pulse width is independent from the transmission distance, namely, $t_{w}=\Theta(1)$. Also, the behavior of the DMC channel differs from that observed in optical communications, where chromatic dispersion causes the pulse width to increase proportionally to the transmission distance, i.e., $t_{w}=\Theta(r)$, at a slower rate than in DMC.

With respect to the pulse energy in DMC, equation (10) shows that it scales as $E_{p}=\Theta(1 / r)$. In wireless EM communications, since there is no signal distortion in a free-space environment, the scalability of the pulse energy is proportional to that of its amplitude, i.e., $E_{p}=\Theta\left(1 / r^{2}\right)$. In this case, DMC using energy detection shows a scalability advantage with respect to wireless EM communications. Moreover, the pulse energy scales better than the pulse amplitude as the transmission distance increases. In other words, an energy detection scheme will allow successful transmissions at longer distances than one based on amplitude detection. Intuitively, the reason of this difference is that, even though the diffusion process severely reduces the amplitude of the transmitted pulses, it also increases 
their width; therefore, the energy of a molecular pulse decreases more slowly than its amplitude as the transmission distance increases.

Finally, we observe in (12) that the pulse duration scales with the transmission distance as $t_{D}=\Theta\left(r^{2}\right)$. As it happened with the pulse width, the signal duration in wireless EM communications is independent of the transmission distance: $t_{D}=\Theta(1)$. In this case, the scalability pattern of the pulse duration matches that observed for the pulse width in the amplitude detection case. Therefore, even though in a typical scenario the pulse duration will be higher than the pulse width (as we have seen in Sec. VI-B), and thus the achievable transmission rate with energy detection will be lower than with amplitude detection, both pulse detection techniques show the same scalability trend in terms of the achievable transmission rate as a function of the distance.

In consequence, both methods, energy detection and amplitude detection, have their own advantages and drawbacks when they are used in DMC. On the one hand, the receiver sensitivity scales better in the energy detection scenario with respect to when amplitude detection is used. On the other hand, amplitude detection will yield in general a higher achievable transmission rate than energy detection, even though they both scale identically with respect to the distance.

This guideline can be observed in Fig. 10, which shows the reception of a molecular pulse for transmission distances of $2 \mu \mathrm{m}$ (left) and $3 \mu \mathrm{m}$ (right). The transmitted molecular pulse consists of $5 \cdot 10^{6}$ molecules released at time $t=0$, and the pulse duration is calculated as the time after which a fraction $\alpha=0.5$ of the total pulse energy is measured by the receiver. On the one hand, in terms of receiver sensitivity, we find that the pulse amplitude $c_{\max }$ at a transmission distance of $r=3 \mu \mathrm{m}$ is reduced by a factor of $70 \%$ with respect to its value for $r=2 \mu \mathrm{m}$, whereas the decrease in the pulse energy $E_{p}$ is much less severe, at $33 \%$. This observation is in agreement with the prediction that energy detection scales better than amplitude detection as the transmission distance increases. On the other hand, the pulse width $t_{w}$ is found to have a lower value than the pulse duration $t_{D}$ in both cases, which confirms that, for a given transmission distance, amplitude detection will allow a higher achievable transmission rate than energy detection in DMC networks.

To summarize, we envisage energy detection to be a more feasible alternative for DMC when the signal attenuation represents the main limitation, i.e., for long transmission distances. Conversely, amplitude detection may prove more useful in scenarios with low signal attenuation, i.e., for short transmission distances, since it will allow nanosystems to communicate information at a higher rate. Table I compares the scalability of the analyzed communication metrics in DMC and wireless

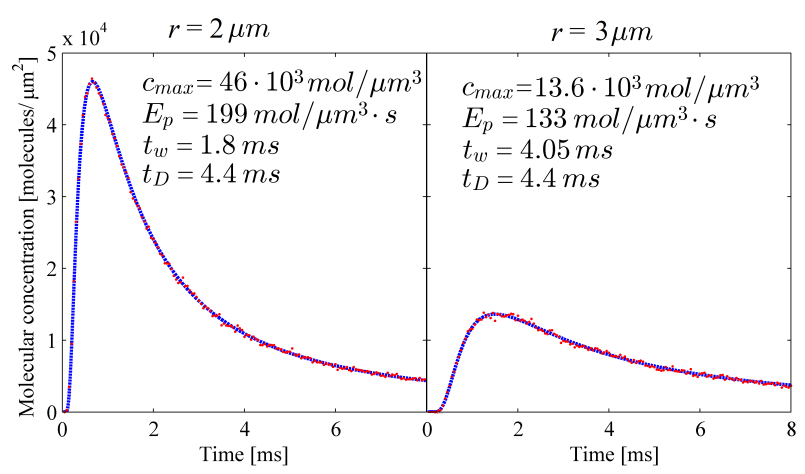

Fig. 10. Molecular concentration as a function of time measured by a receiver located at a distance of $2 \mu \mathrm{m}$ (left) and $3 \mu \mathrm{m}$ (right) from the transmitter. A pulse of $5 \cdot 10^{6}$ molecules is transmitted at time $t=0$. The blue dashed line shows the analytical results and the red dots correspond to simulation results. The impact of the transmission distance on the pulse amplitude $c_{\max }$, the pulse energy $E_{p}$, the pulse width $t_{w}$ and the pulse duration $t_{D}$ can be observed.

TABLE I

COMMUNICATION METRICS IN DMC AND WIRELESS EM COMMUNICATIONS.

\begin{tabular}{l|c|c} 
Metric & DMC & Wireless EM \\
\hline Pulse delay & $\Theta\left(r^{2}\right)$ & $\Theta(r)$ \\
Pulse amplitude & $\Theta\left(1 / r^{3}\right)$ & $\Theta\left(1 / r^{2}\right)$ \\
Pulse width & $\Theta\left(r^{2}\right)$ & $\Theta(1)$ \\
Pulse energy & $\Theta(1 / r)$ & $\Theta\left(1 / r^{2}\right)$ \\
Pulse duration & $\Theta\left(r^{2}\right)$ & $\Theta(1)$
\end{tabular}

EM communications.

\section{CONCLUSION}

In this paper, we have proposed and evaluated the performance of two techniques to detect transmitted signals in DMC networks, namely, amplitude detection and energy detection. First, we have described our envisaged scenario, where molecules emitted by transmitter nanosystems diffuse throughout the medium according to Fick's laws of diffusion.

We adopted a simple pulse-based modulation scheme which we consider specially well suited to the DMC environment. Based on this scheme, and for each of the proposed signal detection techniques, we have identified several metrics which we consider of key importance to evaluate the performance of a DMC system from the communication standpoint. For the amplitude detection case, we have evaluated the pulse delay, the pulse amplitude and the pulse width; whereas for energy detection, we have considered the pulse energy and the pulse duration. We have have obtained analytical expressions for each of these performance metrics and we have validated them by simulation.

Finally, we have outlined the differences in the scalability of the obtained metrics for DMC with respect to their equivalent in wireless EM communications. 
Comparing the scalability of the performance metrics in the amplitude detection and energy detection scenarios, we have concluded that energy detection may be a more feasible alternative when the transmission distances are relatively high, whereas amplitude detection will allow achieving a higher transmission rate in scenarios with low transmission distances.

The future work in this direction might include the biological implementation of the proposed detection mechanisms, and the design of coding schemes and multiple access control protocols for DMC networks. Moreover, the analysis of different sources of molecular noise, such as considering a background molecular concentration or the interference when more than one transmitter is simultaneously emitting molecules, may provide further guidelines for designers of future DMC networks.

\section{ACKNOWLEDGMENT}

This work has been partially supported by the FPU grant of the Spanish Ministry of Education.

\section{REFERENCES}

[1] R. A. Freitas, "Nanotechnology, nanomedicine and nanosurgery." International Journal of Surgery, vol. 3, no. 4, pp. 243-6, 2005.

[2] C. M. J. Pieterse and M. Dicke, "Plant interactions with microbes and insects: from molecular mechanisms to ecology," Trends in plant science, vol. 12, no. 12, pp. 564-9, 2007.

[3] J. Han, J. Fu, and R. B. Schoch, "Molecular sieving using nanofilters: past, present and future," Lab on a chip, vol. 8, no. 1, pp. 23-33, 2008.

[4] I. F. Akyildiz, F. Brunetti, and C. Blázquez, "Nanonetworks: A new communication paradigm," Computer Networks, vol. 52, no. 12 , pp. 2260-2279, 2008.

[5] E. Carafoli, "Calcium signaling: a tale for all seasons." Proc. of the National Academy of Sciences of the United States of America, vol. 99, no. 3, pp. 1115-22, 2002.

[6] T. Nakano, T. Suda, M. J. Moore, R. Egashira, A. Enomoto, K. Ito, and K. Arima, "Molecular communication for nanomachines using intercellular calcium signaling," IEEE Conference on Nanotechnology, vol. 2, pp. 478-481, 2005.

[7] F. Walsh, S. Balasubramaniam, D. Botvich, and W. Donnelly, "Review of communication mechanisms for biological Nano and MEMS devices," in BIONETICS, 2007, pp. 307-312.

[8] M. Pierobon and I. F. Akyildiz, "A physical end-to-end model for molecular communication in nanonetworks," IEEE Journal on Selected Areas in Communications, vol. 28, no. 4, pp. 602-611, 2010.

[9] M. Pierobon, I. F. Akyildiz, and S. Member, "Diffusion-based Noise Analysis for Molecular Communication in Nanonetworks," IEEE Transactions on Signal Processing, vol. 59, no. 6, pp. 2532-2547, 2011.

[10] M. Pierobon and I. F. Akyildiz, "Noise Analysis in LigandBinding Reception for Molecular Communication in Nanonetworks," IEEE Transactions on Signal Processing, vol. 59, no. 9, pp. 4168-4182, 2011.

[11] B. Atakan and O. B. Akan, "On molecular multiple-access, broadcast, and relay channels in nanonetworks," in BIONETICS, 2008.
[12] M. Pierobon and I. F. Akyildiz, "Information Capacity of Diffusion-based Molecular Communication in Nanonetworks," in IEEE INFOCOM Miniconference, vol. 59, no. 4. IEEE, 2011, pp. 602-611.

[13] D. Arifler, "Capacity Analysis of a Diffusion-Based Short-Range Molecular Nano-Communication Channel," Computer Networks, vol. 55 , no. 6 , pp. $1426-1434,2011$.

[14] A. Einolghozati and M. Sardari, "Capacity of diffusion-based molecular communication with ligand receptors," in Information Theory Workshop, 2011.

[15] I. Llatser, E. Alarcón, and M. Pierobon, "Diffusion-based Channel Characterization in Molecular Nanonetworks," in IEEE International Workshop on Molecular and Nanoscale Communications (MoNaCom), 2011, pp. 467-472.

[16] D. E. Clapham, "Calcium signaling," Cell, vol. 131, no. 6, pp. 1047-58, 2007.

[17] I. Karatzas and S. E. Shreve, Brownian Motion and Stochastic Calculus. Springer, 1991.

[18] J. Philibert, "One and a Half Century of Diffusion: Fick, Einstein, before and beyond," Diffusion Fundamentals, vol. 4, no. 6, pp. $1-19,2006$.

[19] T. S. Ursell, "The Diffusion Equation. A Multi-dimensional Tutorial," California Institute of Technology, Pasadena, Tech. Rep., 2007.

[20] H.-Y. Yeh, M. V. Yates, W. Chen, and A. Mulchandani, "Realtime molecular methods to detect infectious viruses." Seminars in cell \& developmental biology, vol. 20, no. 1, pp. 49-54, 2009.

[21] W. H. Bossert and E. O. Wilson, "The analysis of olfactory communication among animals." Journal of theoretical biology, vol. 5, no. 3, pp. 443-69, 1963.

[22] B. S. Donahue and R. Abercrombie, "Free diffusion coefficient of ionic calcium in cytoplasm," Cell Calcium, vol. 8, no. 6, pp. 437-448, 1987.

[23] I. Llatser, N. Garralda, A. Cabellos-aparicio, M. Pierobon, E. Alarcón, and J. Solé-Pareta, "Exploring the Physical Channel of Diffusion-based Molecular Communication by Simulation," in IEEE GLOBECOM, 2011, pp. 566-570.

[24] "NaNoNetworking Center in Catalunya," http://www.n3cat.upc. edu/n3sim.

[25] R. M. Corless, G. H. Gonnet, D. E. G. Hare, D. J. Jeffrey, and D. E. Knuth, "On the Lambert W function," Advances in Computational Mathematics, vol. 5, no. 1, pp. 329-359, 1996.

[26] A. Papoulis, Probability, random variables, and stochastic processes, ser. McGraw-Hill series in electrical engineering. McGraw-Hill, 1984.

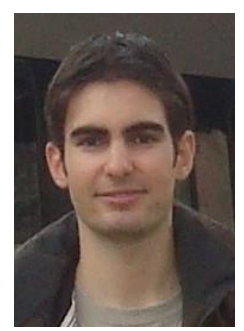

Ignacio Llatser graduated with a double degree in Telecommunication Engineering and Computer Science from the Technical University of Catalonia (UPC) in 2008, where he also received a M.Sc. in Computer Architecture, Networks and Systems in 2011. He is currently pursuing a Ph.D. in Computer Architecture at the Nanonetworking Center in Catalunya (N3Cat) within UPC. He has spent several research stays at the École Polytechnique Fédérale de Lausanne (2008), the Georgia Institute of Technology (2011 and 2013) and the Bergische Universität Wuppertal (2012). His research interests lie in the fields of nanonetworks, molecular communication and graphene-enabled wireless communications. 


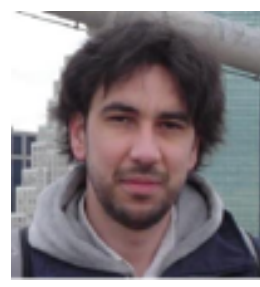

Albert Cabellos-Aparicio received a B.Sc. (2001), M.Sc. (2005) and Ph.D. (2008) degree in Computer Science Engineering from the Technical University of Catalonia (UPC). In September 2005 he became an assistant professor of the Computer Architecture Department and as a researcher in the Broadband Communications Group (http://cba.upc.edu/). In 2010 he joined the Nanonetworking Center in Catalunya (http://www.n3cat.upc.edu) where he is the Scientific Director. $\mathrm{He}$ is an editor of the Elsevier Journal on Nano Computer Network, Computer Networks and member of the Project Management Committee of the LISPmob opensource initiative (http://lispmob.org). He has been a visiting professor at KTH (2012) and MIT (2013). His main research interests are future architectures for the Internet and nanonetworks.

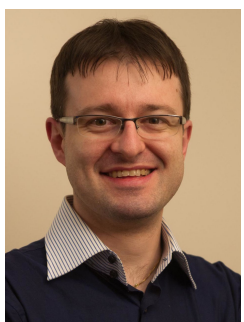

Massimiliano Pierobon is currently a $\mathrm{Ph} . \mathrm{D}$. candidate and graduate research assistant at the Broadband Wireless Networking Laboratory (BWN lab), School of Electrical and Computer Engineering, Georgia Institute of Technology. He received the M. Sc. degree in Telecommunication Engineering from the Politecnico di Milano, Italy, in 2005. During 2006, Massimiliano Pierobon worked as a researcher in the R\&D department of Siemens Carrier Networks, Milan, where he coauthored two filed patents on jitter buffer management. From January 2007 to July 2009 he was a graduate research assistant at the Politecnico di Milano in the fields of signal processing and pattern recognition. In November 2008 Massimiliano Pierobon joined the BWN lab, first as a visiting researcher and, from August 2009, as a Georgia Tech Ph.D. student. His current research interests are in molecular communication theory for nanonetworks, communication engineering applied to intelligent drug delivery systems and bacteriabased network engineering.

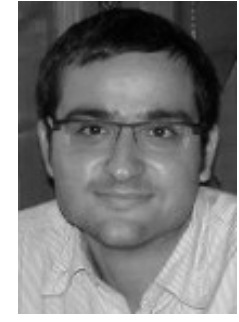

Eduard Alarcón (S'96, M'01), received M.Sc. (national award) and Ph.D. degrees in EE from the Technical University of Catalonia, Barcelona, Spain, in 1995 and 2000, respectively, where he became Associate Professor in 2001, and has been visiting Professor at University of Colorado at Boulder, USA (2003) and KTH Stockholm (2011). He has coauthored more than 250 scientific publications, 4 book chapters and 4 patents, and has been involved in different national, EU and US R\&D projects. Research interests include the areas of on-chip energy management circuits, energy harvesting and wireless energy transfer, and nanocommunications. He was elected IEEE CAS society distinguished lecturer, elected member of the IEEE CAS Board of Governors (2010-2013), recipient of Best paper award at IEEE MWSCAS98, co-editor of 4 journals special issues, 5 conference special sessions, TPC co-chair and TPC member of 15 IEEE conferences, and Associate Editor for IEEE TCAS-I, TCAS-II, JETCAS, JOLPE and Nano Communication Networks. 